\title{
Hunt for the Quark-Gluon Plasma: 20 Years Later
}

\author{
Takeshi Kodama \\ Instituto de Física, Universidade Federal do Rio de Janeiro, Caixa Postal 68528, 21941-972, Rio de Janeiro, RJ, Brasil
}

Received on 22 August, 2003.

\begin{abstract}
We review briefly the recent progress in the search for the quark-gluon plasma (QGP) at CERN SPS and BNL RHIC. Several model analyses and observables, such as hadronic thermal equilibrium, hydrodynamical flow, HBT, jet quenching, etc. which lead to the present conception of the formation of QGP are described.
\end{abstract}

\section{Introduction}

It has been more than two decades since the basic idea of using ultra relativistic heavy ion collisions to create the quarkgluon plasma (QGP) was proposed[1]. Quantum Chromodynamics (QCD) has predicted the possible existence of this new state of matter at very high temperature where quarks and gluons become deconfined and thus play the role of the carriers of the thermal degrees of freedom rather than hadrons. Such states are supposed to be established in the early stage of the Universe before hadronic matter emerges. Thus, we are trying to reproduce the early stage of the Universe in terms of ultrarelativistic heavy ion collisions.

In early times, the expectation of a clear-cut first order phase transition from hadronic matter to QGP attracted many people, and it was hoped that the observation of the signals of such phase transition in hadronic or nuclear interactions would constitute a dramatic confirmation of QCD. So, the basic motivation for the use of heavy ions rather than elementary hadronic collisions is to achieve thermal equilibrium to extract directly thermodynamical signals of the phase transition, such as the identification of latent heat by the construction of caloric curves (entropy density vs. temperature).

Experimentally, heavy ion collisions are more expensive and difficult than hadronic collisions for the same energy. Furthermore, the final states of the system become orders of magnitude more complex due to the corresponding large number of degrees of freedom involved. Therefore, methods of data storage and their analysis also become complex. However, when we want to investigate the thermodynamical signals of a system, we should first establish the thermodynamical equilibrium. This necessarily requires large interaction time and volume. In hadron-hadron collisions, the system seems to be too small to attain the thermal equilibrium. Thus the complexity of heavy ion collision is the price we have to pay.

The relativistic heavy ion collision program started with the incident energy $\sim 1 \mathrm{GeV}$ per nucleon using the Tevatron, in Berkeley, just before the 80's. Subsequently, in Brookhaven and Dubna, fixed target nuclear collisions at relativistic energies have been studied. In the 90's, a heavy ion program with fixed target started at the CERN SPS accelerator. The center of mass energy then was of the order of a few tens of $\mathrm{GeV}$ per nucleon pairs. In the end of the year 2000, the Relativistic Heavy Ion Collider (RHIC) at BNL began to work, with energies of $100-200 \mathrm{GeV}$ at the center of mass, and in the near future (2007) the Large Hadron Collider (LHC) at CERN will be in operation for a heavy ion program in the energy range of a few $\mathrm{TeVs}$.

During these two decades, especially from the experiments realized at SPS and RHIC, a lot of new data have been obtained and is still being accumulated. They revealed the extremely complex nature of the physical processes involved in heavy ion collisions. Instead of the initial naive QGP signals, necessities of more sophisticated observables have been recognized. Although the nature of the transition from the hadronic phase to the QGP phase is still not well understood, the over-all "picture" of relativistic heavy ion collisions is now being configured. In addition to the experimental data, advances in theoretical studies also enriched understandings of the properties of the strong interacting matter[2]. In particular, recent developments in lattice-QCD calculations are very significant[3]. A new issue is the possible existence of a critical point in the $(T, \mu)$ phase diagram[4]. This means that there will be no first order phase transition for baryon-antibaryon symmetric matter. In this short review, we report the present status of the ultrarelativistic heavy ion collision physics, mostly based on the works reported during the last Quark Matter conference at Nantes[5].

\section{Partonic Excitations in the First Hadronic Interactions}

Nowadays, no one raises any serious objections against QCD as the theory of strong interactions and, of course, to the existence of quarks of gluons (it was not so only 30 years ago and ideas of relativistic heavy ion collisions were even out of question). Therefore, when we say "QGP signals", we are not talking about a mere observation of the presence of quark-gluon degrees of freedom, but how they become deconfined and behave as the real carriers of the thermo- 
dynamical energy and momentum. That is, we are talking about dynamical mobilities of quarks and gluons.

First, let us consider the structure of hadrons in terms of quarks and gluons. In the QCD picture, a hadron is a bound state of quarks which may be illustrated as in Fig.1.

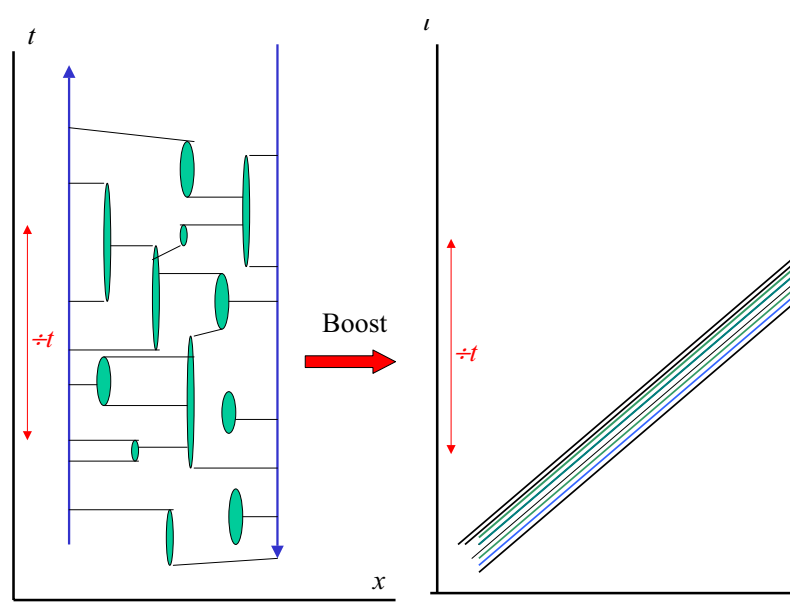

Figure 1. Virtual lines become uncorrelated within a short time interval $\Delta t$ when the hadron is boosted.

In the left-hand side of this figure, a hadron (in this case, a meson) structure is illustrated in terms of two (valence) quark lines where the vertical direction represents the time and horizontal direction the space. Between the two lines many (infinite) virtual bubbles formed by quark-antiquark pairs are connected with gluon exchanges. Suppose we need a certain minimum time interval $\Delta t$ to observe the structure of this hadron. All virtual lines which are shorter than this time interval are strongly correlated so that they are not observable as individual degrees of freedom. Only their average behavior reflects the observed hadron properties. When the hadron is boosted near the speed of light (the right-hand side of the figure), then due to Lorentz dilation of time all these short-living bubbles elongate and the effect of correlation among them becomes irrelevant within the time interval $\Delta t$. In fact, all these virtual quarks and anti-quarks, together with gluons, can be regarded as independent particles within this short interaction time interval. Therefore, it is these "independent particles" that are responsible for the first interactions among high energy hadron collisions. They are called "partons". Then we expect that the number of these partons will increase when the incident energy increases, since for higher energies we can "see" more uncorrelated virtual lines within a given interaction time $\Delta t$. How the number of partons increases as a function of the incident energy depends on the interaction which generates the virtual pairs in a hadron, i.e., depends on the ground state of the system. In practice, this energy (momentum) dependence of the number of partons is observed as the structure functions of a hadron in deep inelastic collisions.

After the first instant of the collision in hadron-hadron collisions, these partons are shot out from their initial configurations and lead to the hadron production in terms of fragmentation of strings. In the case of nucleus-nucleus collisions, a huge number of partons is produced so that if in- teractions among them are sufficiently effective, thermalization of the partonic gas will be attained. This is the QGP. In this sense, the QGP is expected to be formed in high energy nucleus-nucleus collisions as a consequence of "melting" nucleons at high energy density. However, since these partons are nothing but the basic ingredients which constitute the background for the nucleon's proper existence, i.e., the physical vacuum itself, we may also say that the QGP is a melted state of the physical vacuum. In this aspect, the role of colliding heavy ions is to serve as a pressure cooker to melt the vacuum.

\section{Thermal Equilibrium and Relevant Observables}

Unfortunately, the melted vacuum cannot be tasted directly, since any observables are in the form of hadrons far after their formation at the melting point. This is somewhat similar to ask pizza delivery from Rio to Águas de Lindoya. Even if the pizza has nicely melted cheese on it while it is in the oven, it will be deadly cold when we receive it here in Águas de Lindoya (see Fig.2).

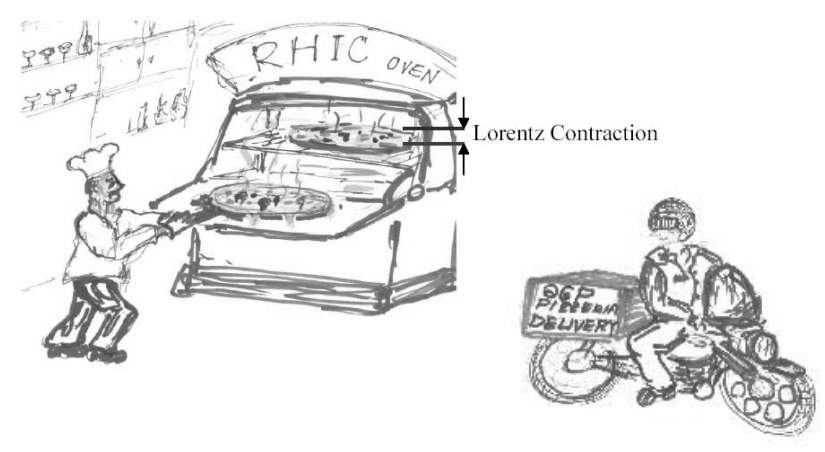

Figure 2. Can we taste the flavor of the melted vacuum?

Therefore, we have to find some way to taste the flavor of melted vacuum from the cold pizza. This is also exactly the case of the modern observational cosmology, where we try to find out signals of the initial melted state of matter just after the Big-Bang in terms of the present-day (cold pizza) signals.

It may be illustrative to classify possible QGP signals into two major categories. One which is related directly or indirectly to the thermodynamical or hydrodynamical properties of the QGP dynamics. The other are the effects of the deconfined quark and gluon gas on the way of propagation of some probe particle in the QGP. In the first category, we may list the particle abundances, especially the strangeness enhancement, the collective flow properties and azimuthal asymmetry parameters, etc. In the second category, we may classify the $J / \psi$ suppression, dilepton production, direct photons and jet quenching. In both cases, there are some intrinsic shortcomings. For example, as we 
mentioned in the Introduction, the concept of thermal equilibrium becomes delicate when we deal with a small system within a short time scale. In a strict sense, we never expect the true thermal equilibrium in nucleus-nucleus collisions. Therefore, the signals of QGP in the first category may be spoiled by this finite size effect. The signals of the second category have the advantage of being free from the necessity of the ideal thermal equilibrium. We just want to verify the formation of finite domains where the quarks and gluons are deconfined. However, this depends on how effectively the hot and dense deconfined quarks and gluons interact with the probing particles and how this process can be distinguished from the one where one has a collection of hadrons instead of the QGP.

In any case, the first thing we have to check is whether we can really use the concept of thermal equilibrium, or resort to states very close to it, in each stage of a relativistic heavy ion collision. To answer this question, several observables are analyzed, namely the transverse momentum distribution, its dependence on particle species, thermal model analyses of particle abundances (ratios), elliptic flow and HBT interferometry, the latter to measure the source size and expansion. One interesting result comes from the thermal model fit to particle abundance ratios[6].

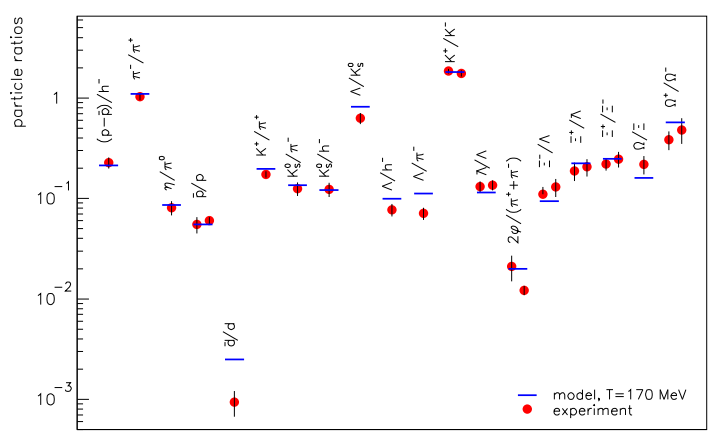

Figure 3. Thermal model fit to the SPS $\mathrm{PbPb}$ collisions. Figure taken from Ref. 6.

In Fig.3, we show the thermal model fit of various particle ratios for the $\mathrm{Pb}+\mathrm{Pb}$ collisions at SPS energy. A similar fit is also obtained for the RHIC $\mathrm{Au}+\mathrm{Au}$ collisions. In these models, it is assumed that there exists a stage where the chemical abundances of every hadron (including resonances) are suddenly frozen, keeping the exact memory of the last instant of chemical equilibrium. Such stage is referred to as "chemical freeze-out" and the observed hadronic abundances are completely determined by the temperature $T$ and the baryonic chemical potential $\mu_{B}$ of the chemical freeze-out[6].

The above picture is equivalent to say that the hadronic gas at the chemical freeze-out is described by a grand canonical ensemble (GC). The fact is that the observed particle ratios are remarkably well reproduced adjusting just two parameters, $T$ and $\mu_{B}$. Although the resulting fits are impressive, it is very difficult to imagine that final hadrons are in a global chemical equilibrium (rapidity distributions of particles, especially those of hyperons, are far from constant). It may well be possible that to have a nice fit only for the chemical abundances do not imply that $T$ and $\mu_{B}$ obtained should correspond to those for a real chemical equilibrium but are just some effective parameters to characterize some average of statistical ensembles corresponding to different physical conditions for chemical freeze-out. In order to clarify this point, we should go at least one step further than the mean values of chemical abundances, such as event-byevent fluctuations and correlations of chemical abundances among different hadrons. In such direction, the distribution of the $K / \pi$ ratio has been measured. In fact, the observed width of this quantity is much larger than that given by a unique grand canonical ensemble[7]. Therefore, the above thermal model does not necessarily prove that there is a uniform chemical equilibrium of the system. Some contaminations of different chemical potentials should be taken into account. Nevertheless, as seen in Fig.4., it is very suggestive to observe that the correlation between the two particle ratios, $\bar{K} / K$ and $\bar{p} / p$ almost agrees with that given by the grand canonical ensemble[8].

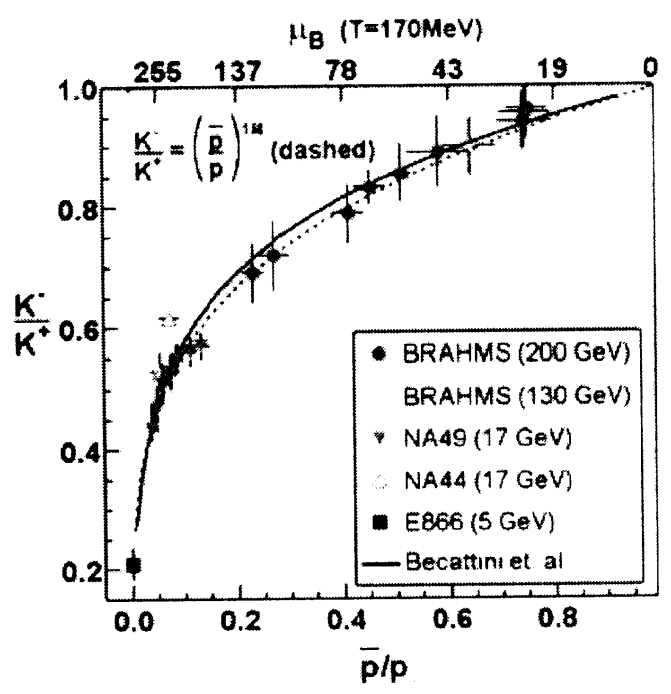

Figure 4. Correlation between particle ratios, $\bar{K} / K$ and $\bar{p} / p$. Figure taken from Ref.8.

In Fig. 5, we show the phase diagram according to the present knowledge. The dashed curve indicates the possible first order phase transition between the hadronic and the deconfined QGP phases with theoretical uncertainty bars from lattice QCD. Data points indicate the chemical freeze-out of various nuclear reactions from AGS, SPS and RHIC. It is interesting to observe that these chemical freeze-out points are on the curve of constant energy per baryon $(\simeq 1.1 \mathrm{GeV})$.

The information about the thermal equilibrium of the final state can also be studied by the transverse momentum distribution of the particles. In Fig. 6 we show the spectra for different hadrons at the central region for $\mathrm{Au}-\mathrm{Au}$ central collisions at RHIC $(\sqrt{s}=200 G e V)[9]$. The inverse slope parameter $T^{*}$ of these spectra is related not only to the temperature but also to the transverse collective flow of the matter from which the hadrons are emitted. Assuming that 
these particles are emitted from an expanding fluid, we can determine from the mass dependence of the inverse slope parameter the temperature and the transverse collective velocity of the fluid. The analysis of STAR data indicates that a consistent fit to these spectra is given by $T_{f} \simeq 100-110$ $\mathrm{MeV}$ with $\beta_{T} \simeq 0.6$. It is interesting to note that a similar fit for the SPS data gives a little bit larger $T_{f} \simeq 120-130$ $\mathrm{MeV}$, although it is a increasing function of the incident energy up to SPS. This may indicate that, at RHIC energy, the transverse expansion of the system becomes dominant, the system enlarges and consequently the freeze-out occurs at a lower temperature.

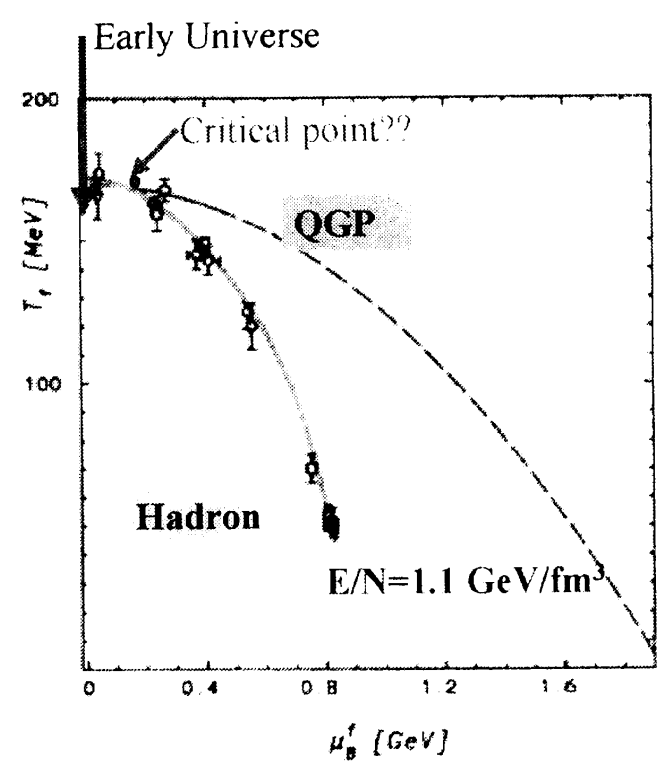

Figure 5. Phase diagram of hadronic and QGP.

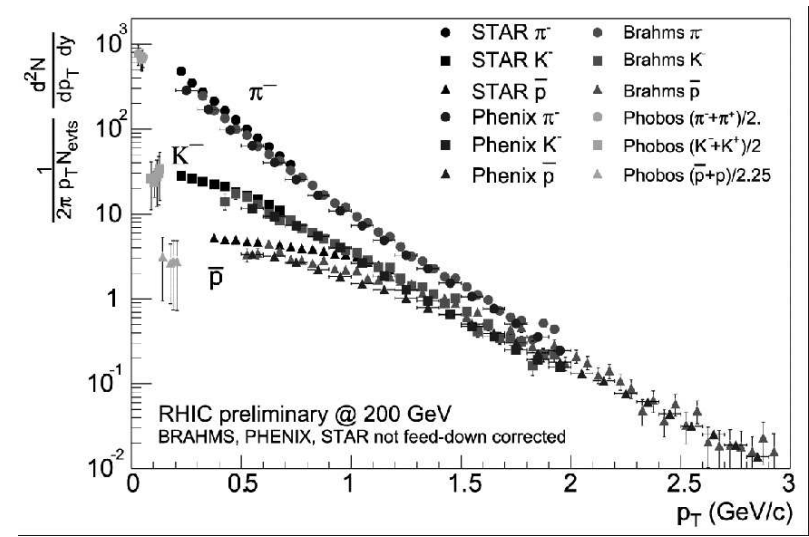

Figure 6. Mass dependence of the transverse momentum distribution. Figure taken from Ref.9.

The temperature above refers to the kinematical freezeout and should be distinguished from the chemical freezeout ( $T_{c f} \simeq 160 \mathrm{MeV}$ for RHIC). This is because the number of hyperons can change only by the process of strangeness annihilation and such processes stop in stages that happen earlier than the thermal scatterings.

\section{Hydrodynamical Description and Related Observables}

As we have seen above, there are several evidences for good thermal and chemical equilibrium of the final hadronic state formed in RHIC collisions. If it is really so, we may consider that the extraction of the QGP properties from the hadronic gas observables shown above would be quite difficult, since the thermal equilibrium in general washes out the information of the initial state. On the other hand, there are some indications which show that this picture of complete hadronic chemical and thermal equilibrium may have some loopholes. Several authors defend the existence of signals (gun-smoke) of QGP explosion from a detailed study of these observables[10]. Various other possible signatures are: entropy increase (due to the release of new degrees of freedom, namely color), strangeness increase (due to enhanced strange quark production and faster equilibration), $J / \psi$ suppression (due to color screening or collision with hard gluons) or enhancement at higher energy (due to recombination of dissociated $c \bar{c}$ pairs), production of leptons and photons (emitted from a thermalized QGP and nearly unaffected by strong interactions), etc.[2]. These signals have been studied extensively in experiments (see for example [11]). More recently, the observed high transverse momentum depletion in $\mathrm{Au}+\mathrm{Au}$ central collisions at RHIC energies (jet quenching) and also its absence in $\mathrm{d}+\mathrm{Au}$ collisions, together with the system-size dependence of mono-jet formation, have been considered as a convincing evidence of the formation of QGP at RHIC[12]. This is the type of signals which are classified in the second category we mentioned in the previous section (probes for the deconfined quarks and gluons) so that they are, in principle, free from the requirement of thermal equilibrium for the QGP state. The basic point is the difference between the energy loss mechanism of parton propagation in the QGP gas and in the hadonic gas. $J / \psi$ suppression signal is also of this type.

As we mentioned before, the thermodynamical signatures are delicate for finite systems. In such cases, a more careful analysis would be necessary to clarify the effect of finiteness and dynamical evolution of the system on these signals[13]. Some authors suggest that the incident energy dependence of several quantities should be studied and they claim that the set of incident energy dependences of particle multiplicity, average transverse momentum and kaon/pion ratio as a whole indicate the appearance of the mixed phase in central collisions at SPS energies[14]. In fact, if we consider one specific signal of thermodynamical nature, there are many factors which may give a similar response of the claimed signal. For example, it is well known that the hadronic final state interaction also works to suppress $J / \psi$ and the system size dependence should be carefully studied to extract the significance of the observed data. Therefore, it is always important to study and grasp the hadronic effects on other proposed signals.

One strategy is then to assume a complete thermal equilibrium is attained locally and study the consequences which 
will serve as the standard to be compared with the observed data. Another strategy, completely opposite, is to assume that the nucleus-nucleus collision is a superposition of hadron-hadron collisions and use the results as the standard. Both studies should be done. One promising approach is to combine the hydrodynamical approach and the final state interactions in terms of the UrQMD model[15]. However, due to the complexity of the calculation and many uncertainties of input data, such as hadronic cross sections, it is not easy to get a simple physical insight for the behavior of the observed quantities. What is important is, obviously, that the model used is well-defined in the sense that every assumption is clearly defined quantitatively. Any systematic deviation of the observed data from these analyses certainly will give important information on the properties of matter.

In the hydrodynamical approach, we assume that the dynamics of the system is described by

$$
\partial_{\mu} T^{\mu \nu}=0
$$

where $T^{\mu \nu}$ is the energy-momentum tensor of the fluid. For the case of a perfect gas, we can write

$$
T^{\mu \nu}=(\varepsilon+p) u^{\mu} u^{\nu}-p g^{\mu \nu},
$$

where $\varepsilon, p$ and $u^{\mu}$ are, respectively, the proper energy density, pressure and four-velocity of the fluid element. Eq.(1) should be complemented by the continuity equations for conserved currents such as the baryon number, and also the equation of state. In the case where there is no conserved charge (pion gas), it is sufficient to give the functional relation between $\varepsilon$ and $p$. When the baryon number is included, then the equation of state may be given by

$$
\varepsilon=\varepsilon(n, s),
$$

where $n$ and $s$ are the baryon number and the entropy densities, respectively. These hydrodynamical equations can also be derived from the action principle[16] and can be solved numerically to give the space-time development for the thermodynamical variables and the fluid velocity $u^{\mu}[17]$. To analyze the physical observables in terms of the hydrodynamical scenario, we have to construct the particle spectra from the hydro solution. In the standard hydrodynamical models, one introduces the concept of freeze-out, which assumes that particle emission occurs on a sharp three-dimensional surface (defined for example by $T(x, y, z, t)=$ constant). Before crossing it, particles have a hydrodynamical behavior, and after they free-stream toward the detectors, keeping memory of the conditions (flow, temperature) of where and when they crossed the three dimensional surface. The Cooper-Frye formula [20] gives the invariant momentum distribution in this case

$$
E d^{3} N / d p^{3}=\int_{\sigma} d \sigma_{\mu} p^{\mu} f(x, p) .
$$

$d \sigma_{\mu}$ is the surface element 4-vector of the freeze out surface $\sigma$ and $f$ the thermal distribution function of the type of particles considered. The space-time dependence comes from those of thermodynamical parameters, such as temperature and chemical potential. This is the formula implicitly used in all standard thermal and hydrodynamical model calculations (see [21] for different approach of hadron production from the thermal gas). In Fig. 7, we show results of hydro calculations compared to the observed particle spectra[19].
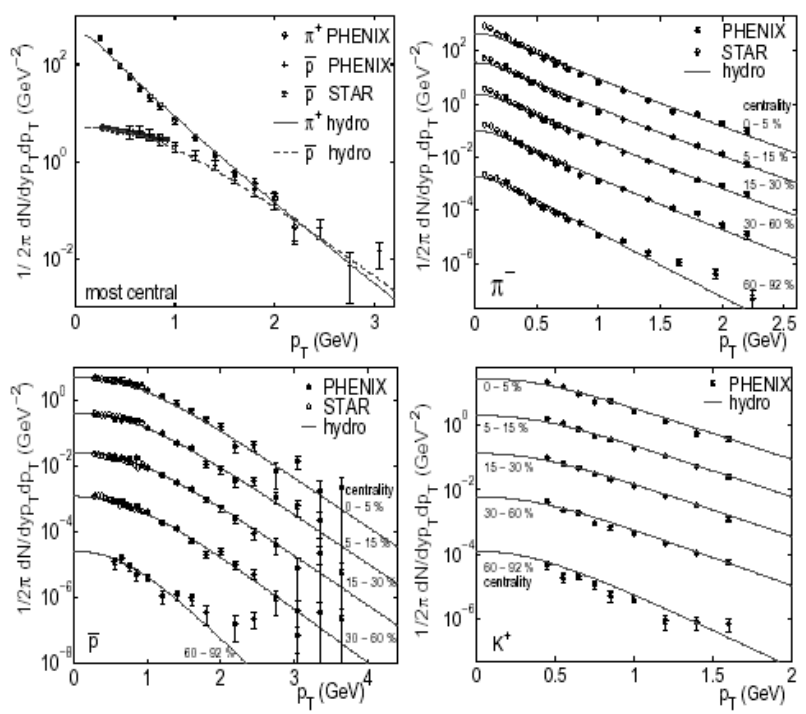

Figure 7. Hydrodynamical calculation for particle spectra and comparison with RHIC data. Figure taken from Ref. 18.

One of the most important observables in the hydrodynamical approach is the flow parameter. In the hydrodynamical picture, for non-central collisions, the anisotropic pressure gradient will result in anisotropic distribution of particles with respect to the reaction plane. Such anisotropy can be expressed by the coefficients of Fourier expansion of the azimuthal distribution of particles. In practice, the determination of these coefficients from the experimental data is not trivial since the reaction plane is not given a priori. In the hydro case, of course, the reaction plane is given from the beginning. The finite positive value of the second coefficient $v_{2}$ is referred to as the elliptic flow and, from the point of view of hydrodynamics, it is sensitive to the initial pressure gradient of the system. In Fig. 8 hydro calculations of the elliptic flow for different equation of states are shown together with the experimental data. In this calculation, the experimental data favor equations of state with the presence of the QGP relative to that given by a pure hadronic gas.

These hydro calculations, together with the other observables, such as pion interferometry (HBT[18]) measurements indicate that the thermal equilibrium of QGP is attained at a very early stage of the nucleus-nucleus collisions[19].

\section{Discussion and Perspectives}

We may summarize the present general picture of the QGP hunting in the relativistic heavy ion collisions.

- Collisional geometry and kinematics are usually under good control (determination of impact parameter, reaction plane, etc.). 
- Hadronic abundances are well described in terms of chemical equilibrium among hadronic resonances, which in turn indicate the temperature and chemical potential of this stage. Temperatures as high as 170 $\mathrm{MeV}$ are achieved in RHIC data.

- Hadronic spectra are consistent with thermal equilibrium and hydrodynamical transverse expansion.

- Hydrodynamical calculations work basically well. This approach indicates the very early equilibration of the partonic gas.

- HBT measurements also indicate a short equilibration time.

- Some indications of non-equilibrium processes. Multi-strangeness enhancement. $J / \psi$ and heavy quark observations.

- Observations of jet-quenching, distribution of monojets, and their dependence on system size effect (jet tomography) indicate the existence of hot and dense deconfined quark and gluon domain.

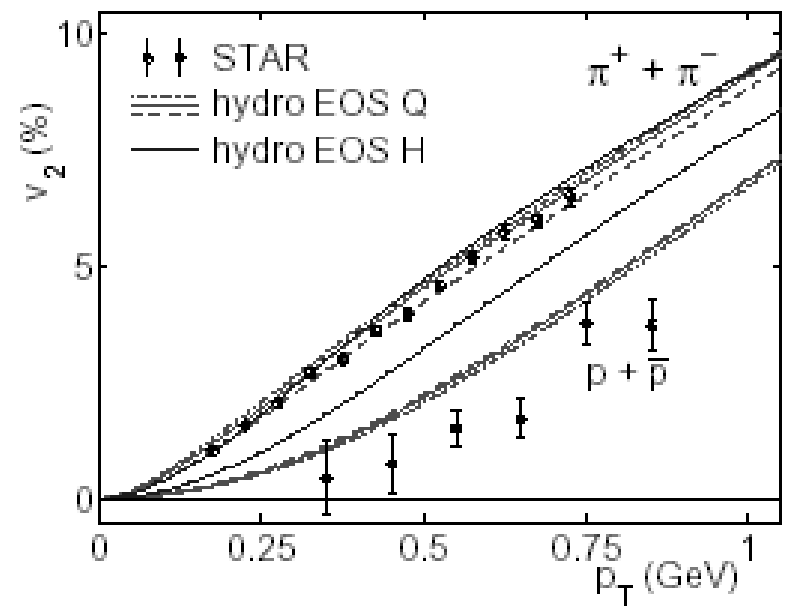

Figure 8. Elliptic flow coefficient $v_{2}$. Figure taken from Ref.18.

The above items strongly indicate that the QGP in relativistic heavy ion collisions was already achieved. However, we still do not know the precise nature of the QGP and its formation dynamics. In the coming LHC experiment (ALICE), observations of the hard components (jets) and of heavy quark production will be the central issue. There, the hydrodynamic description will still become more reliable for bulk dynamics. However, it was pointed out that the effect of even-by-event fluctuations due to the different initial conditions are crucial for quantitative studies of observables[22]. One important point to be reminded with respect to the hydro: while we use the equation of state, hydrodynamical equations are meaningful only when the approximation of local thermal equilibrium is reasonable. On the other hand, as far as Eq.(1) is concerned, it is nothing but the local conservation of energy and momentum density. Therefore, it may be possible that a system which is completely out of local thermal equilibrium can also manifest a flow pattern. In this sense, the study of hydrodynamics coupled to the other degrees of freedom, for example to a chiral field, will be interesting. Studies in this directions are in progress[23].

The author expresses his thanks to F.Grassi, Y.Hama, C.E.Aguiar and E.Fraga for stimulating discussions and kind help. This work was partially supported by FAPERJ, CNPQ and CAPES.

\section{References}

[1] See for example, S. Nagamiya and M. Gyulassy, Adv.Nucl.Phys.13, 201 (1984).

[2] See the recent text book by J. Letessier and J. Rafelski, “Hadrons and Quark Matter", Cambridge Univ. Press 2002.

[3] F. Karsch, Nucl. Phys. A 698, 199 (2002).

[4] Z. Fodor and S. D. Katz, Phys. Lett. B 534, 87 (2002).

[5] See Proceedings of the 16th International Conference on Ultra-Relativistic Nucleus-Nucleus Collisions, (QM02), Nantes, France; 18-24 July, 2002; Edited by H. Gutbrod, J. Aichelin and K. Werner; Nucl. Phys. A715, (2003).

[6] P. Braun-Munzinger, K. Redlich, and J. Stachel, nuclth/0304013

[7] A. Majumder and V. Koch, nucl-th/0305047.

[8] I.G.Bearden, Nucl. Phys. A715, 171 (2003)

[9] T. Ullrich, Nucl. Phys. A715, 399 (2003).

[10] J. Rafelski and J. Letessier, Phys. Rev. Lett. 85, 4695 (2000); Phys. A715, 98 (2003).

[11] J. W. Harris and B. Müller B Ann. Rev. Nucl. Part. Sc. 46, 71 (1996).

[12] S. S. Adler et al. nucl-ex/0306021; J. Adams et al. nuclex/0306024; B.B. Back et al. nucl-ex/0306025; M. Gyulassy, Talk at the 8th International Wigner Symposium

[13] H. Stöcker et al., AIP Conference Proceedings vol 631 p 553 (2001)

[14] M. Gazdzicki and M. Gorenstein, Acta Phys. Polon. B30, 2705 (1999); M. Gazdzicki et al. hep-ph0303041; M. Gazdzicki, hep-ph/0305176.

[15] L. V. Bravina et al. Phys. Rev. C 60, 024904 (1999).

[16] H. T. Elze, Y. Hama, T. Kodama, M. Makler, and J. Rafelski, J. Phys. G 25, 1935 (1999).

[17] C.E. Aguiar, T. Kodama, T. Osada, and Y. Hama, J. Phys. G27, 75 (2001).

[18] see for example, U.A. Wiedermann and U. Heinz, nuclth;9901094.

[19] see for example, P.F. Kolb and U. Heinz, nucl-th/0305084.

[20] F. Cooper and G. Frye, Phys. Rev. D 10, 186 (1976)

[21] F. Grassi, Y. Hama, and T. Kodama, Phys. Lett. B 355, 9 (1955); Z. Phys. C 73, 153 (1966).

[22] C.E. Aguiar, Y. Hama, T. Kodama, and T. Osada, AIP Conf. Proc. 631686 (2003).

[23] C.E. Aguiar, E.S. Fraga, and T. Kodama, nucl-th/0306041 